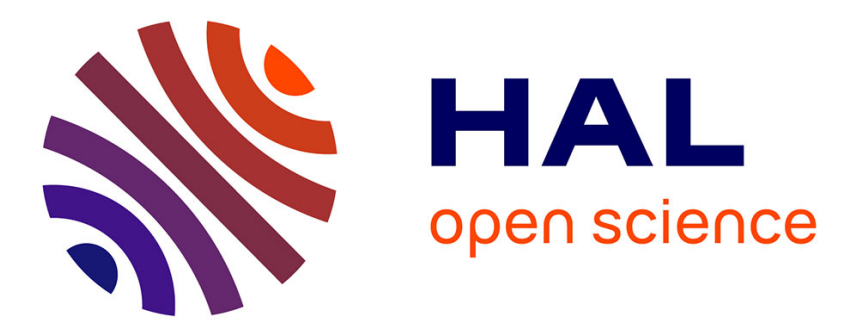

\title{
Concentrations de carbonate de calcium sur les parois des hyphes mycéliens
}

\author{
Gabriel Callot, Daniel Mousain, Claude Plassard
}

\section{To cite this version:}

Gabriel Callot, Daniel Mousain, Claude Plassard. Concentrations de carbonate de calcium sur les parois des hyphes mycéliens. Agronomie, 1985, 5 (2), pp.143-150. hal-00884743

\section{HAL Id: hal-00884743 \\ https://hal.science/hal-00884743}

Submitted on 1 Jan 1985

HAL is a multi-disciplinary open access archive for the deposit and dissemination of scientific research documents, whether they are published or not. The documents may come from teaching and research institutions in France or abroad, or from public or private research centers.
L'archive ouverte pluridisciplinaire HAL, est destinée au dépôt et à la diffusion de documents scientifiques de niveau recherche, publiés ou non, émanant des établissements d'enseignement et de recherche français ou étrangers, des laboratoires publics ou privés. 


\title{
Concentrations de carbonate de calcium sur les parois des hyphes mycéliens
}

\author{
Gabriel CALLOT, Daniel MOUSAIN (*) \& Claude PLASSARD (*) \\ I.N.R.A. Laboratoire de Science du Sol, \\ (*) Laboratoire des Symbiotes des Racines, Place Viala, F 34060 Montpellier Cedex
}

RÉSUMÉ

\begin{abstract}
Après avoir rappelé la structure des hyphes mycéliens, les auteurs mettent l'accent sur les phénomènes d'exsorption (acidification) et d'accumulation des éléments minéraux par les champignons. A partir d'études expérimentales, ils montrent en particulier la possibilité de formation de carbonate de calcium sur les parois des filaments mycéliens. Parallèlement à ces études expérimentales, l'analyse micro-structurale d'échantillons naturels montre l'abondance de ces concentrations minérales dans les sols calcaires et fissures de roches.
\end{abstract}

Mots clés additionnels : Microfissures, minéralogenèse.

\begin{abstract}
Concentration of calcium carbonate on the walls of fungal hyphae.
The structure and function of fungal hyphae is recalled with particular reference to exsorption (acidification) and accumulation of mineral elements. In laboratory experiments, the authors demonstrated the precipitation of calcium carbonate on the outer walls of fungal hyphae. Experiments showed that these and other biochemical transformations could take place very rapidly. In parallel with this experimental study, the microstructure of calcareous soils and rocks has been examined. The role of fungal hyphae in the formation of particular transformations has been clearly shown.
\end{abstract}

Additional key words : Microfissures, mineralogenesis.

\section{INTRODUCTION}

En science du sol, les auteurs insistent sur la solubilisation accélérée des minéraux par les microorganismes (BERTHELIN, 1977). L'action des algues, des champignons et des lichens est particulièrement visible en zones de montagne (ROBERT et al., 1979). En particulier la sécrétion d'acide oxalique par les champignons peut aboutir à la formation d'oxalate de magnésium mis en évidence sur les lichens (WILSON et al., 1980) ou d'oxalate de calcium identifié sur des hyphes de champignons ectomycorhiziens (CROMACK et al., 1979).

En sédimentologie, l'utilisation des techniques de microscopie électronique permet de plus en plus d'identifier diverses figures de concentrations organominérales d'origine microbiologique. KLAPPA (1979) et CALVET (1982) ont pu mettre en évidence l'abondance de filaments calcifiés d'origine fongique, alguaire, bactérienne ou racinaire (poils), dans la zone vadose (avec battement de nappe) de sédiments quaternaires récents de la côte méditerranéenne.
Ces concentrations minérales sont en fait très fréquentes dans les sols où l'activité des mycéliums associés aux racines est intense, particulièrement sous forêt.

L'étude qui suit a pour but de préciser les relations entre ces mycéliums et le calcaire précipité dans les sols.

\section{RAPPEL DE NOTIONS ÉLÉMENTAIRES SUR LES CHAMPIGNONS FILAMENTEUX}

\section{A. Biologie}

Les champignons filamenteux sont des organismes hétérotrophes, saprophytes, parasites ou symbiotes. Beaucoup de saprophytes sont polyphages et vivent dans le sol où ils dégradent les matières organiques. Les symbiotes s'associent à des Thallophytes chlorophylliens (Cyanophytes ou Algues vertes) pour former des lichens, ou à des racines de plantes supérieures en engendrant des organes mixtes, les mycorhizes. Les 
champignons mycorhiziens comprennent aussi tous les intermédiaires entre les symbiotes obligatoires (Boletus, Tuber, Russula, ...) et facultatifs (Hebeloma, Scleroderma, ...). Ils ont souvent un large spectre d'hôtes mais quelques-uns sont associés à un seul genre (cas de Suillus tridentinus et de Lactarius porninsis avec le mélèze).

\section{B. Structure et organisation}

L'appareil végétatif (ou thalle) est généralement formé de filaments ramifiés latéralement (ou hyphes) dont l'ensemble constitue un mycélium. Les hyphes sont des tubes cylindriques chez les champignons supérieurs (Ascomycètes, Basidiomycètes, Fungi Imperfecti) et des siphons non ou très rarement cloisonnés chez les Phycomycètes et les Zygomycètes. Leur diamètre varie le plus souvent de 1-2 $\mu \mathrm{m}$ à 5$10 \mu \mathrm{m}$. Ils possèdent une paroi squelettique, à l'origine pectique puis qui se charge d'une trame microfibrillaire constituée surtout soit de cellulose, soit d'hémicelluloses (glucanes et mannanes) et d'une quantité plus faible de chitine (BARTNICKI-GARCIA, 1968).

La croissance en longueur est strictement localisée à l'apex des filaments (COCHRANE, 1958 ; LARPENT, 1966) $(10 \mu \mathrm{m}$ en moyenne). Le matériel cellulaire synthétisé dans la zone subapicale est transporté à l'apex en voie de croissance et ce transport est activé par la formation des vacuoles dans les zones distales des hyphes (TURIAN, 1969). L'absorption et l'excrétion de substances peuvent se produire, semble-t-il, en toute partie de la paroi de l'hyphe. Le transport des substances s'effectue par mouvement de protoplasme à l'intérieur de l'hyphe préférentiellement des zones âgées vers l'apex en croissance du fillament (SCHUTTE, 1956).

Le champignon est capable, par des processus d'agrégation et de différenciation d'hyphes, d'édifier de véritables organes dont les plus importants dans la pédogenèse sont : les structures végétatives agrégées.

Deux types d'agrégats mycéliens peuvent pénétrer dans le sol sur de plus grandes distances que les hyphes individuels : les cordons mycéliens et les rhizomorphes. Les premiers sont des agrégations progressives et ramifiées d'hyphes qui s'édifient autour d'un hyphe parental (BUTLER, 1966) (fig. 1). Ils se distinguent des rhizomorphes qui sont des structures unidirectionnelles issues de centres initiateurs, ayant une croissance apicale autonome et dont l'apex peut être hautement structuré (Armillaria mellea) et comporter un méristème sensu stricto (Sphaerostilbe) (LOISEAU, comm. pers., 1973). Il existe cependant des intermédiaires entre ces deux schémas d'organisation.

Quelques auteurs seulement ont étudié les cordons mycéliens des champignons ectomycorhiziens (SCHRAMM, 1966 ; BowEN, 1968, 19:33, ...) : par exemple, les cordons de Rhizopogon luteolus peuvent pénétrer dans le sol jusqu'à $3,5 \mathrm{~cm}$ à partir des ectomycorhizes de Pinus radiata ; ces cordons ont un diamètre variant de 15 à $50 \mu \mathrm{m}$ avec des hyphes individuels s'en échappant sur une longueur de 60 à $350 \mu \mathrm{m}$ et sur environ $40 \mu \mathrm{m}$ de part et d'autre du cordon (fig. $1 \mathrm{c}$ ) (BOWEN, 1968). Cette structure serait plus efficace du point de vue de l'exploitation du sol que celle des rhi-

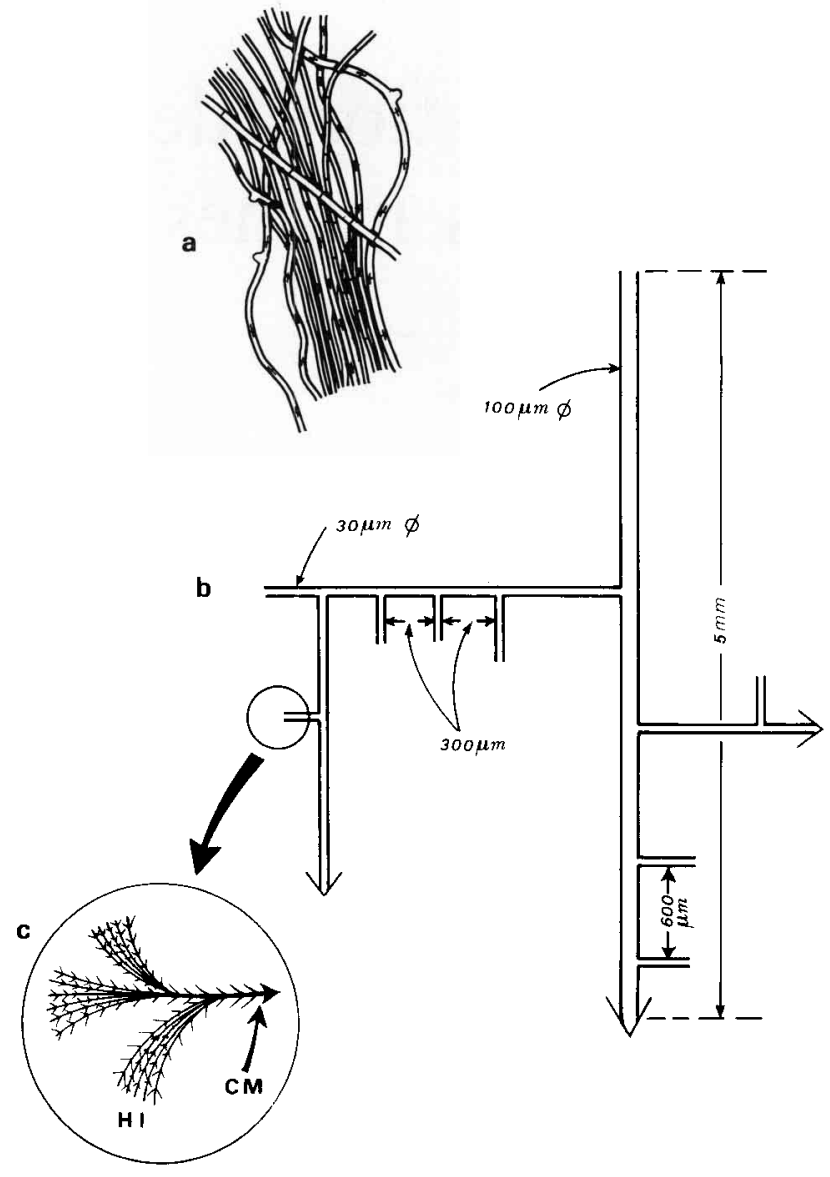

Figure 1

Organisation et structure des cordons mycéliens.

a) Aspect général d'un cordon mycélien de Basidiomycète: les hyphes sont accolés mais conservent leur individualité.

b) Schéma de la ramification d'un cordon mycélien. Exemple d'un cordon mycélien de Rhizopogon luteolus rattaché à des mycorhizes de Pinus radiata (d'après BOWEN, 1973).

c) Détail de l'extrémité d'une ramification.

C.M. : cordon mycélien (15-30 $\mu m$ de diamètre)

H.I. : hyphes individuels (2-4 $\mu m$ de diamètre) développés sur une distance de 60-350 $\mathrm{m}$ et 40 $\mathrm{m}$ de part et d'autre du cordon.

Organisation and structure of mycelial strand.

a) General aspect of mycelial strand of basidiomycete : hyphae are aggregated but preserve their individuality.

b) Diagram of ramification of mycelial strand. Example with mycelial strand of Rhizopogon luteolus connected with mycorrhizas of Pinus radiata (after BOWEN, 1973).

c) Detail of the extremity of a ramification.

C.M. : mycelial strand (15 to $30 \mu \mathrm{m}$ diameter),

H.I. : individual hyphae (2-4 $\mu \mathrm{m}$ diameter) developed on 60-350 $\mu \mathrm{m}$ length and $40 \mu \mathrm{m}$ (lateral).

zomorphes tels que ceux d' $A$. mellea qui présentent une surface lisse et très peu d'hyphes individuels (GARRETT, 1970). SKINNER \& BOWEN (non publié, 1972) ont montré que l'absorption d'éléments nutritifs se produit sur la plus grande part de la longueur du cordon mycélien. Au contraire, les rhizomorphes structurés et subérisés d' $A$. mellea absorbent les phosphates et chlorures principalement au niveau des parties apicales non subérisées (MORRISON, BOWEN, non publié, 1970).

Le rôle des cordons mycéliens apparaît essentiel dans les sols pauvres, notamment vis-à-vis des ions qui diffusent faiblement : il accrô̂t le volume de sol 
exploité par le système " plante-mycorhizes », système économique car l'énergie nécessaire à la croissance du champignon dans le sol est considérablement inférieure à celle qui est requise pour la production de racines explorant le même volume de sol (BJÖRKMAN, 1949). Ainsi, le poids de matière fraîche d'un cordon d'une longueur égale à $22,8 \mathrm{~mm}$ est de $0,125 \mathrm{mg}$, valeur environ 10 fois moindre que celle d'une même longueur de racines courtes non infectées de $P$. radiata (BOWEN, 1968).

L'importance de la colonisation des horizons superficiels de certains sols par les hyphes mycéliens est illustrée par NicHOLAS et al. (1965) : après observation de sections de podzol, ces auteurs ont estimé entre $1,34 \mathrm{~m}$ et $15 \mathrm{~m}$ par $\mathrm{ml}$ de sol, la longueur moyenne des hyphes. Par ailleurs, dans une région tempérée humide et jusqu'à $15 \mathrm{~cm}$ de profondeur, les champignons vivants pourraient rassembler $8.10^{16}$ individus et $2000 \mathrm{~kg}$ de matière sèche $(0,1 \mathrm{p}$. 100) pour 1 ha de sol (BUOL et al., 1973).

\section{Accumulation et excrétion d'ions chez les champi- gnons}

- Les champignons filamenteux accumulent particulièrement les phosphates.

L'étude des champignons mycorhiziens indique que 70 à 90 p. 100 du phosphate accumulé par Rhizopogon luteolus et Suillus luteus cultivés sur une solution contenant du $\mathrm{H}_{2} \mathrm{PO}_{4}^{-} 0,03 \mathrm{mM}$, se trouvent dans un pool non métabolique (BOWEN, non publié, 1966). La teneur en $\mathrm{P}$ total du mycélium de Pisolithus tinctorius atteint 1,1 p. 100 de la matière sèche après $21 \mathrm{j}$ de culture sur milieu comprenant du $\mathrm{H}_{2} \mathrm{PO}_{4}^{-} 2,5 \mathrm{mM}$ (MoU. SAIN \& SALSAC, 1984).

Le manteau fongique des ectomycorhizes joue un rôle essentiel dans l'accumulation du phosphore : 90 p. 100 du phosphate absorbé à partir de solutions faiblement concentrées en cet élément se trouvent dans le manteau et 10 p. 100 dans le " cœur " de la mycorhize (HARLEY \& MC CREADY, 1952 a et b). Le stockage s'effectue sous forme d'orthophosphate, de polyphosphates inorganiques et de phosphates organiques. Les polyphosphates représentent entre 30 et 45 p. 100 du $\mathrm{P}$ accumulé dans le manteau (HARLEY \& MC CREADY, 1981) et sont associés à des granules dans les vacuoles des hyphes des champignons symbiotes (ASHFORD et al., 1975 ; STRULlu et al., 1981). Les hyphes de Glomus mossae associés à Trifolium pratense présentent aussi des granules vacuolaires (taille : 0,1 à $0,3 \mu \mathrm{m}$ ) dont les teneurs en $\mathrm{P}$ et $\mathrm{Ca}$ sont respectivement 5 fois et 6 à 7 fois plus élevées que celles du contenu des vacuoles dépourvues de granules. Par la microanalyse, il a été démontré que les vacuoles des champignons contiennent des polyanions non diffusibles associés à $\mathrm{Ca}^{2+}, \mathrm{K}^{+}$et $\mathrm{Mg}^{2+}$ (STRullu et al., 1981).

- Le zinc, le fer et le sulfate peuvent aussi être accumulés mais en faibles quantités dans les ectomycorhizes des pins et du hêtre (SKINNER et al., non publié, 1972 ; MORRISON, 1962).

- Des études réalisées avec $P$. tinctorius cultivé in vitro sur des milieux de concentration croissante en $\mathrm{Ca}^{++}$montrent que ce champignon accumule du $\mathrm{Ca}^{+}+$dans ses « tissus » (MOUSAIN \& SALSAC, 1984).
L'analyse directe à la microsonde électronique sur le mycélium végétatif et les rhizomorphes de Sphaerostilbe repens indique que le calcium se localiserait préférentiellement au niveau des parois (BOTTON et al., 1980). L'accumulation du calcium peut également se produire dans la vacuole, dans les granules de polyphosphate (STRULLU et al., 1981).

- L'accumulation d'acides organiques, en particulier l'acide oxalique, est fréquente chez les champignons, notamment sur milieu nitrique (CROMACK et al., 1979) comme sur les hyphes du manteau d'ectomycorhizes diverses (FROIDEVAUX \& KÄLIN, 1981 ; MALAJCZUK \& CROMACK, 1982).

- En dehors de l'excrétion d'acide oxalique postulée précédemment, l'exsorption de protons $\left(\mathrm{H}^{+}\right)$par les champignons en culture isolée est régulièrement observée, notamment sur milieu ammoniacal (KRUPA \& BRÄNSTRÖM, 1974 ; SALSAC et al., 1982). Ce mécanisme d'exsorption des protons est ainsi capable d'acidifier très rapidement le milieu : des baisses de 2 unités $\mathrm{pH}$ en $48 \mathrm{~h}$ sont fréquentes en conditions expérimentales (où une solution d'un volume de $90 \mathrm{ml}$ à $\mathrm{pH} 5$ passe rapidement à $\mathrm{pH} 2,9$ en présence d'hyphes mycéliens en croissance). En milieu nitrique, l'exsorption de $\mathrm{H}^{+}$est en grande partie compensée par les processus d'alcalinisation liés à l'absorption et à la réduction du nitrate (RAVEN·\& SMITH, 1976).

\section{CALCITISATION EXPÉRIMENTALE D'HYPHES MYCÉLIENS}

\section{A. Description du dispositif expérimental}

La technique expérimentale utilisée pour obtenir rapidement des racines mycorhizées a pu être employée pour suivre l'évolution et la transformation progressive d'un réseau mycélien au cours de son développement. L'observation sous loupe binoculaire et en microscopie électronique à balayage a permis de suivre l'état de la surface des filaments mycéliens.

Le matériel utilisé est constitué par des échantillons de racines de pin maritime (Pinus pinaster), mycorhizé en conditions contrôlées par Pisolithus tinctorius (cf. Planche IA).

Les semis de pins sont effectués sur un mélange $(\mathrm{v} / \mathrm{v})$ de sable siliceux et de vermiculite, préalablement stérilisé. L'inoculation de ces jeunes pins (âgés de 4 à 5 mois) est effectuée d'après la méthode de FORTIN et al. (1980) légèrement modifiée. Chaque plant, non axénique, est glissé dans un sac de polyéthylène $(15 \mathrm{~cm} \times 28 \mathrm{~cm})$ contenant une feuille de papier filtre stérilisée.

L'alimentation hydrique du plant est assurée par l'intermédiaire d'un tube de verre à travers lequel $15 \mathrm{ml}$ d'eau distillée sont apportés régulièrement dans le fond du sachet. La remontée capillaire dans la feuille de papier filtre assure ainsi à la plante mycorhizée une alimentation en eau suffisante pour assurer son développement.

Plusieurs implants mycéliens gélosés (b), calibrés (diamètre $8 \mathrm{~mm}$ ), sont disposés contre le papier filtre à proximité des racines courtes du pin susceptibles d'être mycorhizées. Ces implants gélosés sont obtenus selon la technique décrite par FORTIN et al. (1980) : le mycélium de $P$. tinctorius est cultivé in vitro en boîte de Petri sur gélose nutritive (milieu Melin-Norkrans 
modifié - «MNM »; MARX, 1969). A partir de ces cultures, on découpe à l'emporte-pièce de nombreux implants que l'on transfère ensuite à nouveau sur " MNM » pendant $8 \mathrm{j}$ environ afin de sélectionner le mycélium le plus actif pour l'inoculation des plants. Après l'inoculation, chaque sac est fermé par 2 attaches et suspendu sur un portoir. Les plants sont maintenus à l'obscurité à $28{ }^{\circ} \mathrm{C}$ pendant $24 \mathrm{~h}$ et transférés ensuite en chambre climatisée (photopériode : 16/24, température de jour : $25{ }^{\circ} \mathrm{C}$; température de nuit : $22{ }^{\circ} \mathrm{C}$; énergie lumineuse ; $20000 \mathrm{erg} . \mathrm{cm}^{-2} \cdot \mathrm{s}^{-1}$ ). Le niveau de la solution est réajusté tous les $5 \mathrm{j}$ environ par de l'eau du robinet.

\section{B. Précipitations des cristaux de calcite au niveau des hyphes mycéliens}

Les observations en microscopie électronique effectuées un mois après l'inoculation nous ont permis d'observer, sur les filaments et cordons mycéliens, diverses formes de cristaux. Ces derniers ont été identifiés en microanalyse (dosage de calcium), d'après leurs formes cristallines et leur réactivité aux vapeurs d'acide chlorhydrique dilué : les cristaux d'exalate de calcium ne font pas effervescence, alors que les cristaux de carbonate de calcium sont très réactifs.
La formation de cristaux d'oxalate de calcium (Planche I, cliché 2), fréquemment observée dans le milieu naturel sur les réseaux d'hyphes de champignons et signalée par CROMACK et al. (1979), FROIDEvaUX \& KÄlin (1981), MalajCZUK \& CROMACK (1982), n'est pas surprenante.

Effectivement, les champignons sécrètent une quantité importante d'acide oxalique, tout particulièrement lorsqu'ils ont été alimentés avec une forte concentration en glucose (milieu gélosé initial) avec une bonne aération du milieu et un $\mathrm{pH}$ initial relativement élevé (BURNETT, 1976). Cet acide oxalique réagit alors avec le calcium présent dans le milieu pour former de l'oxalate de calcium.

Par contre, d'autres filaments mycéliens sont entourés de cristaux de carbonate de calcium (planche I, cliché 3). La formation expérimentale de ces cristaux par les hyphes de champignons ne semble pas avoir été signalée jusqu'ici alors que de nombreux auteurs ont déjà écrit diverses formes de calcification de filaments fongiques ou alguaires dans les sédiments (KLAPPA, 1979 ; KAHLE, 1977 ; KOBLUK \& RISK, 1977 ; etc...).

D'après les auteurs précédents, cette précipitation de carbonate pourrait être induite par des composés organiques de la couche externe mucilagineuse, dans des micro-environnements à forte concentration en calcium et à $\mathrm{pH}$ élevé.

\section{PLANCHE}

Formation de cristaux de carbonate de calcium sur des filaments mycéliens

\section{A. ÉTUDE EXPÉRIMENTALE}

Mycélium de Pisolithus tinctorius et mycorhize de jeune pin (Pinus pinaster).

a - Culture mycélienne en boîte de Petri sur gélose nutritive.

b - Implant mycélien isolé.

c - Sac de polyéthylène avec papier filtre posé sur une paroi.

d - Racine courte mycorhizée.

e - Racine séminale.

Clichés:

1. Voile mycélien, enveloppant les mycorhizes développées sur les coiffes des racines courtes de pin. (R-racine).

2. Cordon mycélien recouvert de cristaux d oxalate de calcium (Ox).

3. Cristaux de carbonate de calcium (Ca) développés sur filaments mycéliens (C.m).

\section{B. SURFACE DE MICROFISSURE DANS CALCAIRE DUR}

Coupe dans un galet calcaire (de $7 \mathrm{~cm}$ de diamètre) selon plan de microfissure de quelque $10 \mu \mathrm{m}$ de largeur, avec cordons et filaments mycéliens $(\mathrm{C} . \mathrm{m})$ reliés à la racine $(\mathrm{R})$ et empreintes d'anciens filaments calcitisés (C.c). Clichés :

4-5. Réseau d’hyphes lisses (C.m), et altérés (C.c) recouverts de cristaux de carbonate de calcium.

PLATE I

Formation of calcite crystal on mycelial filaments

\section{A. EXPERIMENTAL CONTROL :}

Mycelium of Pisolithus tinctorius and mycorrhizas of young pine (Pinus pinaster).

$a-$ Mycelial culture in Petri dish with nutrient agar.

$b-$ Mycelial fragment isolated.

c - Polythene bag with filter paper in contact on a wall.

$d$ - Short root with mycorrhizas.

$e-$ Seminal root.

Photo :

1. Manile of short mycorrhizal roots of pine. (R-root).

2. Accumulation of calcium oxalate $(O x)$ on a mycelial strand.

3. Calcium carbonate $(\mathrm{Ca})$ formed on mycelial hyphae $(\mathrm{Cm})$.

\section{B. MICROCRACKS OF COMPACT LIMESTONE :}

Section in a calcareous pebble $(7 \mathrm{~cm}$ diameter) along a microcrack of $10 \mu \mathrm{m}$ wide with strings and mycelial hyphae (C.m) impression of calcified filaments (C.c).

Photos :

4-5. Aggregated hyphae with glossy (C.m) and altered (C.c.) walls covered with crystals of calcium carbonate. 
A- Etude expérimentale
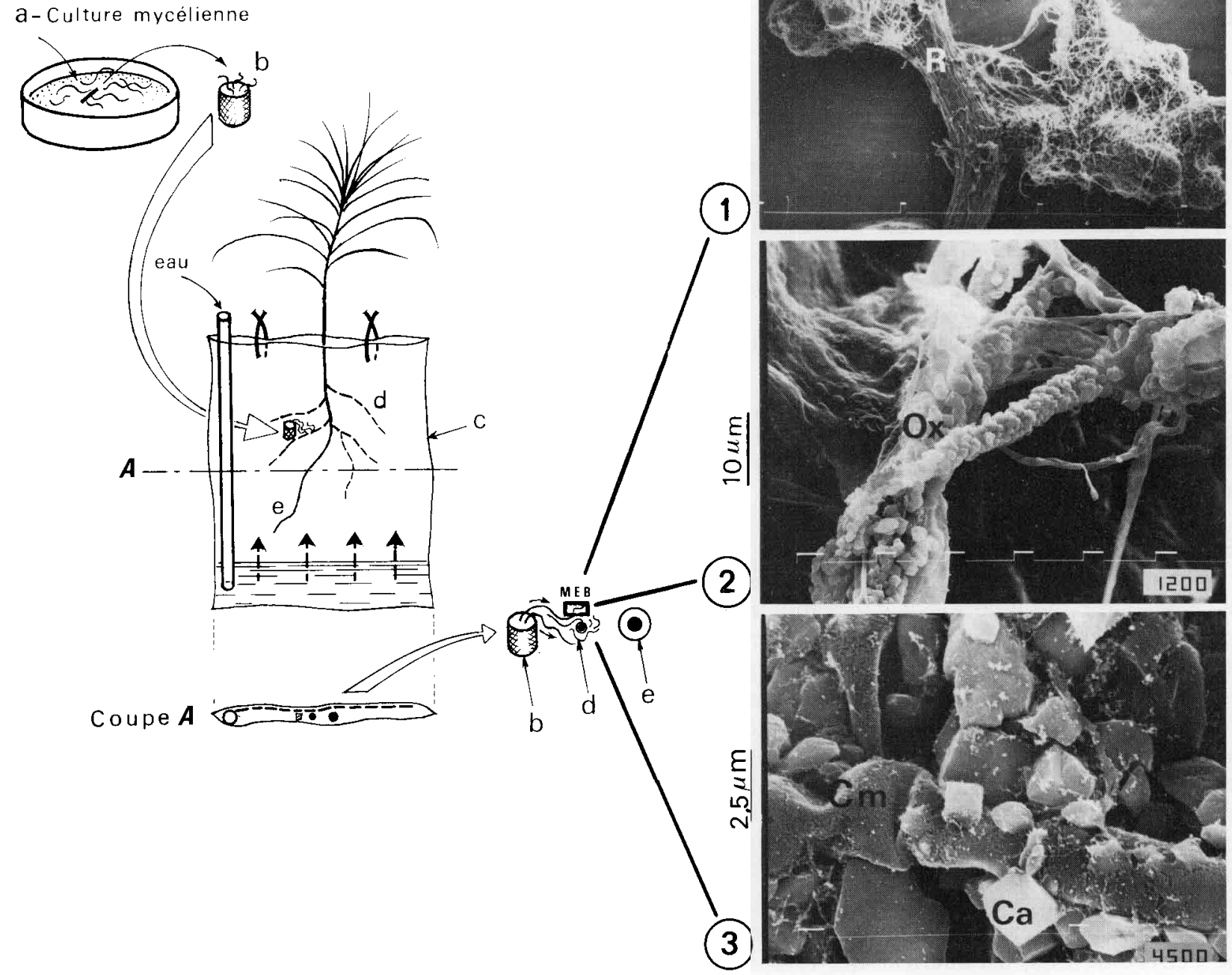

B-Surface de microfissure dans calcaire dur
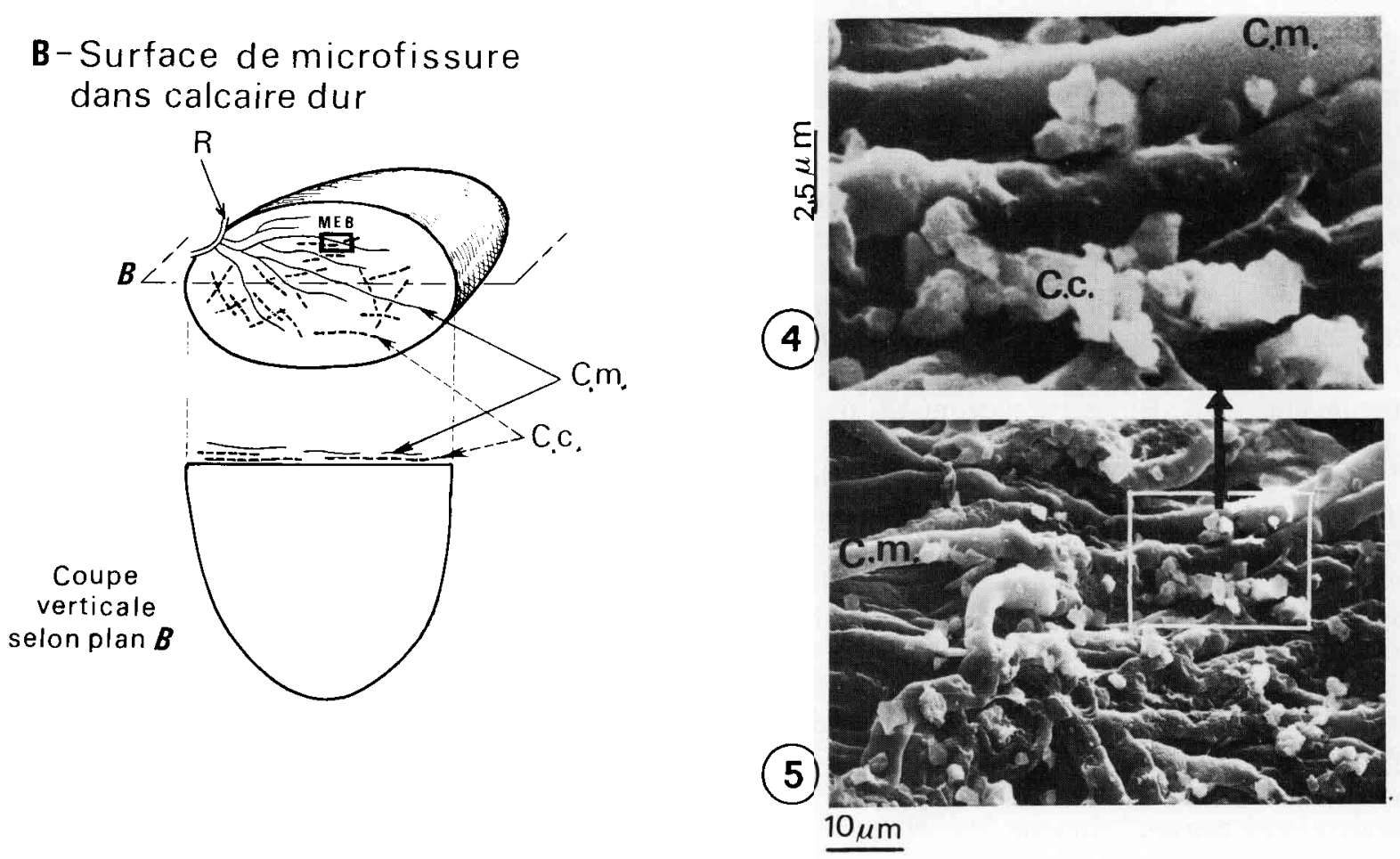
La formation de cristaux de carbonate de calcium sur les parois des hyphes de champignons est également très fréquente dans les sols et les fissures de roches (cf $\S I V)$.

\section{ALTÉRATION MYCÉLIENNE DANS LES MICROFISSURES DE ROCHES CALCAIRES}

De nombreuses figures de " dissolution » de roches, susceptibles d'apparaître à l'œil comme une transformation des structures pétrographiques originelles, résultent le plus souvent de figures de concentrations calcaires sous le contrôle des hyphes mycéliens. L'étude de microfissures de cailloux calcaires prélevés dans un sol sous forêt de pin laisse apparaître des cordons et des hyphes mycéliens (cf planche IB) superposés à des concentrations cristallines issues elles-mêmes d'anciens réseaux mycéliens calcitisés.

L'observation en microscopie électronique laisse apparaître la présence de cristaux de carbonate de calcium identifiés par leur réactivité aux vapeurs d'acide chlorhydrique, sur les filaments mycéliens (clichés 4 , 5).

En conditions expérimentales comme dans le milieu naturel (micro-fissures), les hyphes mycéliens sont donc susceptibles de favoriser la précipitation de cristaux de calcite sur leurs parois. Ils participent ainsi à la transformation progressive des carbonates dans le sol et dans les fissures des roches. Ces concentrations de calcaire fin (micritique), d'origine biologique, s'effectuent à l'extérieur du filament mycélien lorsque l'organisme est encore vivant, phénomène d'incrustation cristalline diffuse sur les parois (ef. cliché 2, planche I). Au cours de la calcitisation progressive, le phénomène affecte la totalité de l'hyphe et peut entraîner la coalescence des hyphes calcitisés comme l'a montré CALVET (1982). La croissance des cristaux de calcite peut s'effectuer aussi à l'intérieur des filaments lorsque l'hyphe est mort (KLAPPA, 1979) et aboutir à la calcitisation totale des structures mycéliennes.

Le modèle d'altération microfissurale des roches calcaires, par les hyphes mycéliens, représenté sur la figure 2, fait apparaître l'aspect simultané des processus de dissolution et de précipitation des carbonates. Les phénomènes de dissolution se développent dans l'espace au cours de l'exploration et de la croissance du système mycélien, suite à l'acidification des milieux par exsorption de protons $\left(\mathrm{H}^{+}\right)$, alors que la précipitation des carbonates s'effectue au cours du temps, sur des hyphes, semble-t-il, plus âgés. Des études expérimentales ultérieures préciseront ces mécanismes et surtout l'origine du calcium.

La vitesse d'évolution du système dépend des conditions du milieu et des climats. Les conditions sèches favorisent la concentration des sels, donc les précipitations, alors que les conditions humides et les fortes teneurs en matière organique accélèrent l'activité biologique, donc la dissolution des sédirnents.

Les phénomènes de précipitation cristalline sur les parois des hyphes dépendent des conditions de milieu (hydrique et thermique), de l'activité des systèmes mycéliens, des solutions circulant dans les fissures. Ces dernières peuvent contenir des éléments absents de la roche encaissante (parois des fissures), ce qui
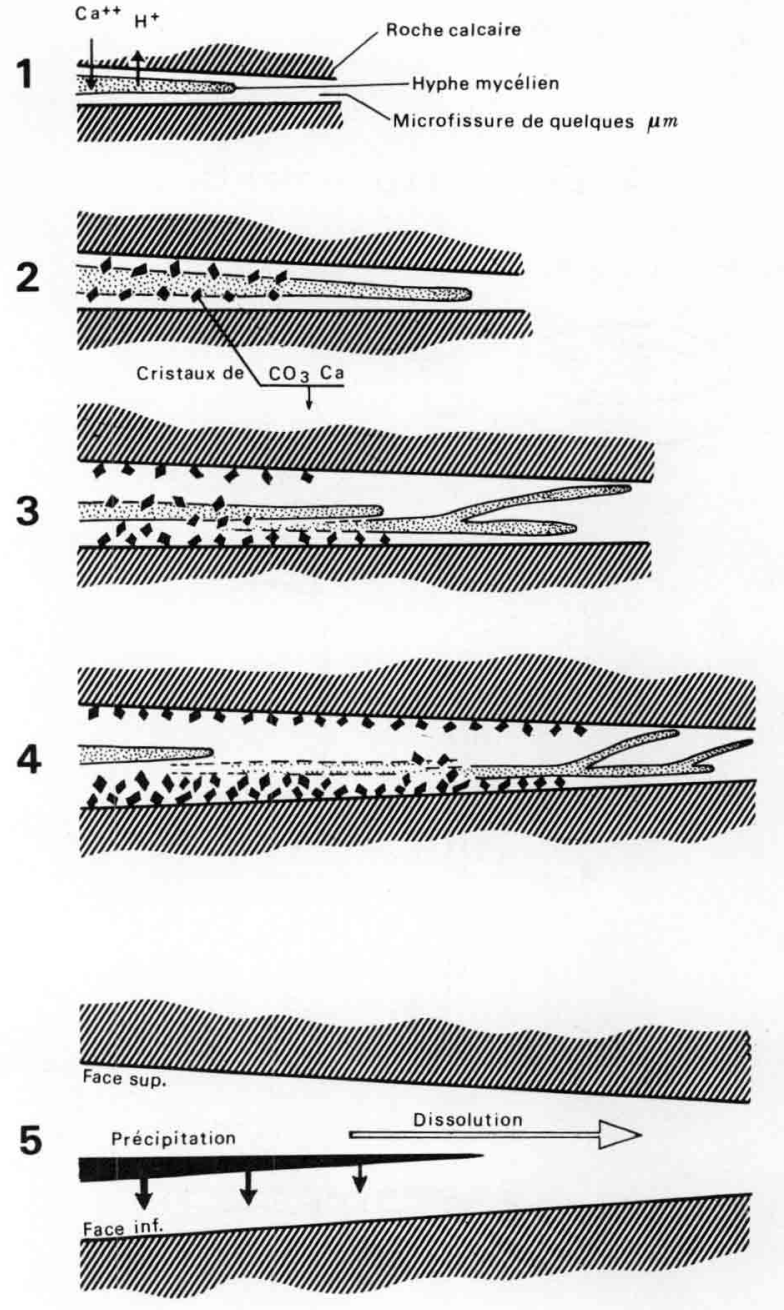

Figure 2

Modèle d'altération microfissurale d'une roche calcaire par les hyphes mycéliens.

1. Phase de dissolution avec hyphes mycéliens en croissance.

2. Croissance des cristaux de calcite sur les parois des hyphes plus âgés.

3. Coalescence d'hyphes calcitisés.

4. Cimentation des cristaux sur les parois des microfissures, plus intense à la face inférieure.

5. Bilan des processus de dissolution/précipitation à caractère biogéo-chimique.

Model of the weathering of a limestone microcrack by fungal hyphae.

1. Dissolution phase during hyphal growth.

2. Calcite crystal growth on the older hyphal walls.

3. Fusion of calcified filaments.

4. Cementation of calcite crystals to microcrack walls especially on the lower surface.

5. Evaluation of biogeochemical process with a dissolution/precipitation mechanism.

explique ainsi les possibilités de précipitation de carbonate de calcium dans les microfissures de roches métamorphiques non calcaires.

\section{CONCLUSION}

Jusqu'alors les processus de transferts minéraux sous l'activité des champignons avaient été très peu abordés. Nos observations micro-structurales au niveau du terrain, comme les premiers résutats d'études expérimentales semblent montrer que les processus 
de transfert «fungo-géo-chimiques » sont très importants dans les sols et dans l'altération des roches.

En effet, il semblerait que les hyphes de champignons soient particulièrement bien adaptés pour participer activement à ces transferts. Ces micro-organismes constitués de filaments de quelques $\mu \mathrm{m}^{2}$ de section puisent leur énergie (sucres et sources carbonées) dans les structures organiques mortes ou vivantes. Ils sécrètent des acides organiques qui contribuent activement à la dissolution des minéraux présents dans le milieu. Mais lorsque les conditions de milieu changent (dessication), les hyphes peuvent alors concentrer les minéraux qu'ils ont dissous pour construire de nouvelles structures critallines (fig. 3). Ces concentrations minérales fongiques, susceptibles de se produire rapidement, confèrent à l'espèce un haut degré de résistance. Ces processus contribuent ainsi activement à la transformation des microstructures du sol et des roches.

Dans les exemples cités, nous avons insisté particulièrement sur ces phénomènes, en milieu calcaire. Mais ces concentrations minérales d'origine mycélienne sont également fréquentes en milieu acide ou des transferts analogues se produisent avec la silice.

A côté des incidences pédogénétiques et minéralogiques évidentes, l'étude de transformations organominérales sous le contrôle de l'activité des hyphes mycéliens pourrait apporter de précieuses données sur le fonctionnement des systèmes symbiotiques dans les milieux naturels et aider à une meilleure compréhension des problèmes de pathologie végétale.

Recu le 10 octobre 1983. Accepté le 28 septembre 1984.

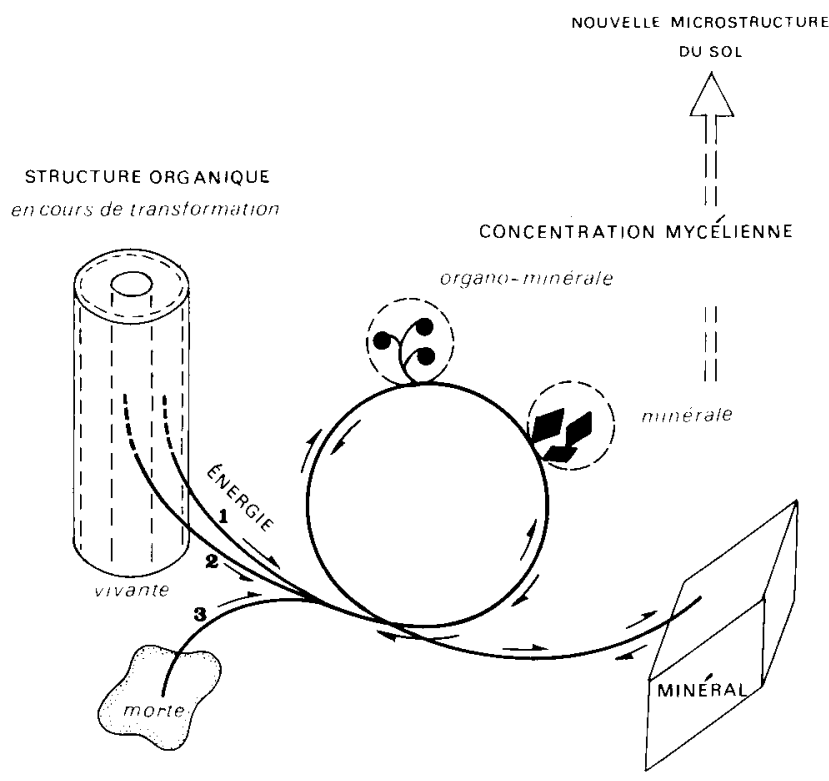

Figure 3

Transformation des microstructures du sol et des roches par l'activité des hyphes mycéliens.

Transformation of soil and rock microstructures by the activity of fungal hyphae.

1. ectomycorhiziens

2. endomycorhiziens

3. saprophytes

1. ectomycorrhizal fungi

2. endomycorrhizal fungi

3. saprophytic fungi

\section{REMERCIEMENTS}

Nous tenons à remercier L. SALSAC, Directeur du Laboratoire des Symbiotes des Racines de l'I.N.R.A. (Montpellier), qui a bien voulu procéder à l'analyse critique du manuscrit.

\section{RÉFÉRENCES BIBLIOGRAPHIQUES}

Ashford A. E., Ling-Lee M., Chilvers G. A., 1975. Polyphosphate in eucalyptus mycorrhizas : a cytochemical demonstration. New Phytol., 74, 447-453.

Bartnicki-Garcia S., 1968. Cell wall chemistry, morphogenesis and taxonomy in fungi. Ann. Rev. Microbiol., 22, 87-108.

Berthelin J., 1977. Quelques aspects des mécanismes de transformation des minéraux des sols par les microorganismes hétérotrophes. AFES, Sci. Sol, I, 13-24.

Björkman E., 1949. The ecological significance of the ectotrophic mycorrhizal association in forest tress. Sver. Bot. Tidskr., 43, p. 223.

Botton B., Fourcy A., Bossy J. P., 1980. Localisation cellulaire du calcium chez le champignon ascomycète Sphaerostilbe repens par analyse directe à la microsonde électronique. Can. J. Bot., 58, 2395-2401.

Bowen G. D., 1968. Phosphate uptake by mycorrhizas and uninfected roots of Pinus radiata, in relation to root distribution. Trans. Int. Congr. Soil Sci. 9th, 2, 219-228.

Bowen G. D., 1973. Mineral nutrition of ectomycorrhizae, 151205 ; in G. C. Marks et T. T. Kozlowski : " Ectomvcorrhizae Their Ecology and Physiology ». Academic Press Inc., N-Y and London, $444 \mathrm{p}$.

Buol S. W., Hofe F. D., Mc Cracken R. J., 1973. Soil Genesis and Classification. The lowa State University Press, $360 \mathrm{p}$.

Burnett J. H., 1976. Fundamentals of Mycology. Edward Arnold, $2^{\mathrm{e}}$ Ed., $673 \mathrm{p}$.
Buttler G. M., 1966. Vegetative structures in the fungi, 83-112. In G. C. Ainsworth \& A. S. Sussman : "The Fungi, 2 ". Academic Press, N. Y. 805 p.

Calvet F., 1982. Constructive micrite envelope developed in vadose continental environment, in pleistocene eolianites of Mallorca (Spain). Acta Geol. Hisp. (in press).

Cochrane V. W., 1958. Physiology of fungi. John Wiley and Sons Inc., New York. Chapman and Hall Limited, London, 524 p.

Cromack K., Sollins P., Todd R. L., Fogec R., Todd A. W., Fender W. M., Crossley M. E., Crossley D. A., 1977. The role of oxalic acid and bicarbonate in calcium cycling by fungi and bacteria : some possible implications for soil animals. Ecol. Bull. (Stockholm), 25, 246-252.

Cromack K., Sollins P., Graustein W. C., Speidel K., Todd A. W., Spycher G., Li C. Y., Todd R. L., 1979. Calcium oxalate accumulation and soil weathering in mats of the hypogeous fungus, Hysterangium crassum. Soil Biol. Biochem., 11, 463-468.

Fortin J. A., Piché Y., Lalonde M., 1980. Technique for the observation of early morphological changes during ectomycorrhiza formation. Can. J. Bot., 58 (3), 361-365.

Froidevaux L., Kälin I., 1981. Accumulation d'oxalate de calcium dans les nodules du champignon mycorhizien Hebeloma crustuliniforme: importance du phénomène pour la nutrition de l'arbre. Schweiz. Z. Forstwes., 132, 339-344.

Garrett S. D., 1970. « Pathogenic Root-Infecting Fungi ». Camb. Univ. Press, London and New York, $294^{\circ}$ p. 
Harley J. L., Mc Cready C. C., 1952 a. The uptake of phosphate by excised mycorrhizal roots of the beech. II. Distribution of phosphorus between host and fungus. New Phytol., 51, 56-64.

Harley J. L., Mc Cready C. C., 1952 b. The uptake of phosphate by excised mycorrhizal roots of the beech. III. The effect of the fungal sheath on the availability of phosphate to the core. New Phytol., 51, 342-348.

Harley J. L., Mc Cready C. C., 1981. Phosphate accumulation in Fagus mycorrhizas. New Phytol., 89, 75-80.

Kahle C. F., 1977. Origin of subaerial Holocene calcareous crusts : role of algae, fungi and sparmicritisation. Sedimentology, 24, 413-435.

Klappa C. F., 1979. Calcified filaments in quaternary calcretes organo-mineral interactions in the subaerial vadose environment J. sédiment Petrol., 49 (3), 955-968.

Kobluk D. R., Risk M. J., 1977. Calcitisation of exposed filaments of endolithic algae, micrite envelope formation and sediment production. J. sediment Petrol., 47 (2), 517-528.

Krupa S., Bränström G., 1974. Studies of the nitrogen metabolism in ectomycorrhizae : II. Free and bound aminoacids in the mycorrhizal fungus Boletus variegatus, in the root systems of Pinus silvestris and during their association. Physiol. Plant., 31, 279-283.

Larpent J. P., 1966. Caractères et déterminisme des corrélations d'inhibition dans le mycélium jeune de quelques champignons. Ann. Sci. Nat., 7, 130

Malajczuk N., Cromack K. (Jr), 1982. Accumulation of calcium oxalate in the mantle of ectomycorrhizal roots of Pinus radiata and Eucalyptus marginata. New Phytol., 92, 527-531.

Marx D. H., 1969. The influence of ectotrophic mycorrhizal fungi on the resistance of pine roots to pathogenic infections. I. Antagonism of mycorrhizal fungi to root pathogenic fungi and soil bacteria. Phytopathology, 59, 153-163.

Morrison T. M., 1962. Uptake of sulphur by mycorrhizal plants. New Phytol., 61, p. 21.
Mousain D., Salsac L., 1982. Nutrition phosphatée et activités phosphatases acides des symbiotes ectomycorhiziens cultivés isolément ou en association. "Les Mvcorhizes : biologie ef utilisation", Dijon, 5-6 mai 1982, Colloque INRA n ${ }^{\circ} 13,87-100$

Mousain D., Salsac L., 1984. Effet du phosphate et du calcium sur la croissance et l'accumulation ionique chez les champignons ectomycorhiziens en culture in vitro. Can. J. Bot. (sous presse).

Nicholas D. P., Parkinson D., Burges N. A., 1965. Studies of fungi in a podzol. II. Application of the soil-sectioning technique to the study of amounts of fungal mycelium in the soil. J. Soil Sci., $16(2)$, 260-269.

Raven J. A., Smith F. A., 1976. Nitrogen assimilation and transport in vascular land plants in relation to intracellular $\mathrm{pH}$ regulation. New Phytol., 76, 415-431.

Robert M., Eyralde J., Berrier J., Pelisonnier C., 1979. Illustration du rôle des êtres vivants dans l'altération et la pédogenèse à l'étage alpin et subalpin. Ann. Univ. Savoie, Tome 4, p. 23-25.

Salsac L., Mention M., Plassard C., Mousain D., 1982. Données sur la nutrition azotée des champignons ectomycorhiziens. "Les Mvcorhizes : biologie et utilisation", Dijon 5-6 mai 1982. Colloque INRA $n^{\circ} 13,129-140$.

Schramm J. R., 1966. Plant colonization studies on black wastes from anthracite mining in Pennsylvania. Am. Philosoph. Soc., 56 $194 \mathrm{p}$.

Schutte K. H., 1956. Translocation in the fungi. New Phytol., 55, 164-182.

Strullu D. G., Gourret J. P., Garrec J. P., 1981. Microanalyse des granules vacuolaires des ectomycorhizes, endomycorhizes et endomycothalles. Physiol. Vég., 19, 367-378.

Turian G., 1969. Différenciation fongique. Masson, Paris.

Wilson M. J., Jones D., Russel J. D., 1980. Glushinskite, a naturally occurring magnesium oxalate. Mineral. Mag., 43, 837-840. 